\title{
Present and Future Directions of Basic Research on Polymeric Materials
}

\author{
Norbert M. BIKALES \\ Division of Materials Research, National Science Foundation, \\ Washington, DC 20550, U.S.A.
}

(Received August 17, 1986)

\begin{abstract}
Polymer science is in the midst of profound changes in direction. Historically, the foundation of polymer science has been primarily chemistry, i.e., the preparation of polymers and physical-chemical studies of their properties. At the present time, the frontiers of the science are being pushed increasingly toward condensed-matter physics and materials science. One reason for this trend is, undoubtedly, the intellectual nourishment that polymer scientists are finding in these fertile fields which, themselves, are undergoing some great changes of their own. Another, of perhaps even greater potential importance, is the discovery by many scientists with little prior interest in polymers that these materials offer an intellectually challenging area of research. A still further impetus comes from the demands of dynamic "high-technology" industries for new materials with new properties. Eventually, bridges will also be built to the biological sciences. All of these changes are occurring at a time when the ever-increasing power of the instrumentation and computers available to the researcher is providing new insights. Together, these forces are reshaping the way in which basic research on polymeric materials will be conducted in the future.
\end{abstract}

KEY WORDS Polymer Science / Chemistry / Physics / Materials Science / Biological Sciences / Basic Research / History of Polymer Science / Instrumentation / High-Technology Industries /

This 2nd SPSJ International Polymer Conference has as its theme "Specialty Polymers for High Performance." It is designed to cover in depth certain topics of great interest to contemporary polymer science, namely high-performance polymers, advanced composites, conductive polymers, the photochemistry of polymers, and polymers for hightechnology applications. Each of the papers represents a significant, tangible advance in the field. Most academic basic research, in Japan as well as in the Unites States, is carried out in this way: by individual researchers, or small groups of researchers (e.g., a professor with several students and postdoctoral fellows). The publications that result from such an effort generally constitute small, but nevertheless very significant, steps in the forward march of science.
Each of the these individual efforts can be further grouped into subareas of science. In the NSF Polymers Program, the subareas of basic polymer science that we are funding at present are listed in Table I, where the percentage of the Program's total funding allocated to each of these subareas in 1986 is also shown. The Engineering Directorate and other parts of NSF provide additional funding for subareas of polymer research not covered by the Polymers Program.

This paper presents the author's view of the general directions in which the field of polymer science as a whole is moving, and of the forces that are changing the way polymer science is being carried out. It does not present in any detail the diverse research being carried out by the thousands of polymer scientists throughout the world (among which are over seven 
Table I. Subareas of polymer science in NSF Polymers Program (1986)

\begin{tabular}{lr}
\hline & $\%$ \\
\hline Morphology and blends & 23.8 \\
Synthesis, reactions & 23.5 \\
Solution properties & 17.9 \\
Molecular dynamics & 10.3 \\
Characterization & 7.7 \\
Mechanical properties and composites & 6.6 \\
Electro-optical properties & 4.7 \\
Surfaces and interfaces & 4.5 \\
Others & 1.0 \\
\cline { 2 - 2 } & 100.0 \\
\hline
\end{tabular}

hundred professors in American universities).

Before we begin this journey into the future, it is instructive to learn from the past. The wonderful book by Professor Herbert Morawetz, Polymers: The Origins and Growth of a Science, ${ }^{1}$ traces the intellectual roots and development of our science. Contemporary polymer science grew out of chemistry: all the great pioneers - Staudinger, Mark, Sakurada, Carothers, Champetier, Flory, Kargin, Marvel, Melville, and others - were trained as chemists. Some of these (like Marvel) were mainly interested in synthesis, others (like Mark) primarily in physical properties, and still others (like Flory) primarily in theory. The scope of most, however, encompassed several of these aspects, e.g., synthesis as well as physical properties of the resulting polymers. The work of the polymer chemist thus emerged as fitting neither purely organic chemistry, nor purely physical chemistry, nor purely any of the other traditional subdivisions of chemistry. [Some people have even viewed it, incorrectly, as an applied art.] This key distinction makes it difficult - even a half-century after Staudinger's work - for polymer chemists to fit easily into traditional chemistry departments in universities, and causes an undesirable isolation of polymer chemistry from the remainder of chemistry. This point was made with great emphasis in the report issued by the US Academy of Sciences in $1981 .^{2}$

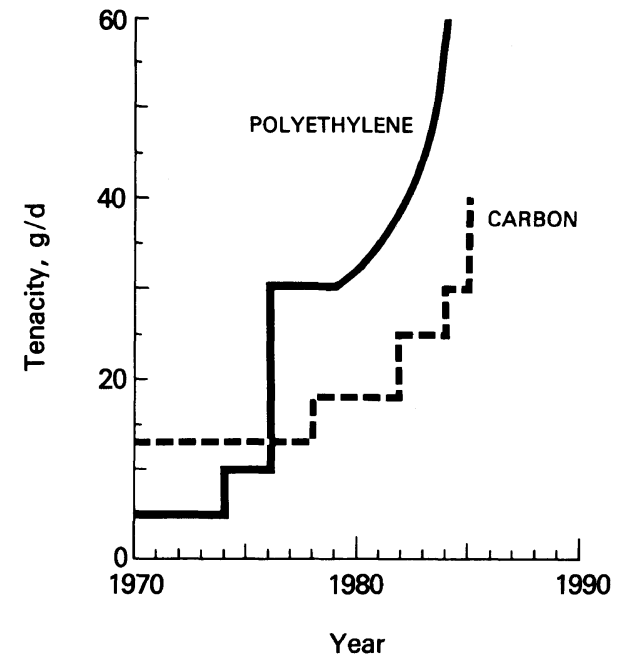

Figure 1. Maximum tenacity achieved in experimental fibers of carbon and polyethylene as a function of time. Courtesy Paul Smith (du Pont).

It should be noted that physicists have also, until recently, shunned polymers, but probably for different reasons: their complexity, and the attraction of biophysics and molecular biology.

But if polymer science has not really achieved full acceptance in some quarters, it has nevertheless immensely flourished. The level of polymer science has been remarkably high, and has had a major impact on science and technology as a whole. In the past few years, polymer scientists have reported such important achievements as synthetic selfassembling systems with remarkable morphologies, ${ }^{3}$ "living" cationic polymerization, ${ }^{4,5}$ polymers approaching the theoretical limit in strength $^{6}$ (see Figure 1), and the direct measurement of adsorptive forces on inorganic substrates. $^{7}$ These are but a few examples of the vigor of contemporary polymer science (taken primarily from subareas of the field not covered at this Conference). The creation and development of specialized departments of polymer science in several universities; the large number of scientific journals, books, meetings and symposia; and the rapidly increasing membership in our scientific socie- 
ties (e.g., 8241 members in 1985 vs. 4274 in 1975 for the Polymer Division of the American Chemical Society) are still other undeniable signs of an astonishing vitality.

Yet, in the midst of this vitality - nourished by the inventiveness and energy of polymer scientists - there are emerging signs that polymer science, as we know it today, is also being reshaped by powerful forces that are essentially external to it:

the dramatic advances being made in other fields of science;

the enormous power of the new instrumentation;

the demands for materials by the "hightechnology" industries.

Let us examine each of these.

\section{THE IMPACT OF ADVANCES IN OTHER FIELDS OF SCIENCE}

We are in the midst of one of the most dynamic periods in the history of science; for example, the number of scientists active today is far greater than at any other time in history, and we are witnessing an astonishing growth in the acquisition of new knowledge. (It has been estimated that there are some 750000 scientists and engineers in the United States working in research and development.) Inevitably, the advances in other fields of science are leaving their impact on polymer science; this is particularly the case with physics.

\section{Physics}

Physicists, who traditionally have played only a secondary role in polymer science, are becoming increasingly active as sources of new ideas and deeper insight into polymer problems as well as active participants, themselves. Table II is a compilation of new concepts and techniques that physicists are contributing to polymer science. ${ }^{8}$

There can be no question that de Gennes' book Scaling Concepts in Polymer Physics ${ }^{9}$ is the most influential since Flory's Principles of Polymer Chemistry. ${ }^{10}$ De Gennes' ideas originate directly from the mainsteam of physics. The same may be said for the profound advances in conductive polymers by Heeger, Schrieffer and others, and the fundamental concepts of the behavior of macromolecules being contributed by Edwards and Doi. ${ }^{8}$ One of the most exciting aspects of current research

Table II. Some ideas from (and to) physics

\begin{tabular}{lll}
\multicolumn{1}{c}{ Physics } & & \multicolumn{1}{c}{ Polymer science } \\
\hline Magnetism & At present \\
$\begin{array}{l}\text { Renormalization group theory } \\
\text { Scaling concepts }\end{array}$ & $\rightarrow$ & $\begin{array}{l}\text { Polymer behavior } \\
\text { Properties of concentrated solutions } \\
\text { Self-diffusion }\end{array}$ \\
Percolation & & Gelation \\
Solitons & $\rightarrow$ & Conductivity in polymers \\
Low dimensionality & $\leftarrow$ & Polymer conformation \\
Low dimensionality & $\rightarrow$ & Langmuir-Blodgett films \\
& In future \\
Low-temperature physics & $\rightarrow$ & Behavior near 0K \\
Ultrahigh pressures & $\rightarrow$ & Phase transitions \\
Superconductivity & $\rightarrow$ & Polymeric superconductors \\
Superlattices & $\rightarrow$ & New designed morphologies \\
Epitaxy & $\rightarrow$ & Layered polymers \\
Fractals & $\rightarrow$ & "Treeing" in plastics \\
New theories & $\rightleftarrows$ & Model polymers \\
Molecular electronics & $\leftarrow$ & Polymer structure and morphology \\
\hline
\end{tabular}


is the growing interplay of theoretical physics with experimental polymer science, and the greater rigor and predictive power resulting from this confrontation.

There are already over forty physics professors in American universities working on polymers. In my opinion, the physicists' role will increase still further in the future, as their ideas become better understood by polymer scientists and even more physicists take up the difficult challenges of complex, polymeric systems.

\section{Chemistry}

There is a perceptible change among American chemists in their attitude toward polymer science. Some "traditional" chemists are already seeking ways to apply their special expertise to the study of polymers, and others are taking the first, tentative steps in that direction. $^{2,11}$ At the same time, several prestigious universities are now seeking to expand, or begin, teaching and research in polymers. The National Science Foundation is nurturing this trend by paying special at- tention to the borderline of chemistry and polymer science; we call this interface materials chemistry.

Table III gives examples of what the traditional subfields of chemistry are contributing to polymer chemistry. A notable recent event is the synthesis of the highly branched polymers known as dendritic (or starburst) polymers ${ }^{12}$ or arborols. ${ }^{13}$ The table illustrates that the flow of ideas also goes from polymer science to chemistry!

\section{Biological Sciences}

From the beginning, there has been a clear link between the study of polymers - synthetic macromolecules - and biological macromolecules; e.g., cellulose, natural rubber, and proteins were among the first macromolecules studied. ${ }^{1}$ However, as molecular biology blossomed and became ever more specialized, that link weakened considerably so that, today, the two fields are quite separate.

Table IV shows some of the new insights that polymer scientists have obtained from the biologists. Here, the interactions are still

Table III. Some ideas from (and to) chemistry

\begin{tabular}{|c|c|c|}
\hline Chemistry & & Polymer science \\
\hline & \multicolumn{2}{|c|}{ At present } \\
\hline Organic chemistry & $\rightarrow$ & $\begin{array}{l}\text { Group-transfer polymerization, new reactions at surfaces, } \\
\text { crown ether polymers }\end{array}$ \\
\hline Organic chemistry & $\leftarrow$ & Polymeric reagents \\
\hline Organometallic chemistry ? & & Olefin metathesis mechanism \\
\hline Inorganic chemistry $\}$ & $\rightarrow$ & $\left\{(\mathrm{SN})_{x}\right.$, polyphosphazenes \\
\hline Physical chemistry & & $\int$ Quasi-elastic light scattering \\
\hline Analytical chemistry & $\rightarrow$ & Excimer fluorescence \\
\hline Solid-state chemistry & $\rightarrow$ & "Stacked" conductive polymers, liquid-crystal behavior \\
\hline \multirow[t]{2}{*}{ Phase-transfer catalysis } & $\leftarrow$ & Interfacial polymerization \\
\hline & \multicolumn{2}{|c|}{ In future } \\
\hline Organic chemistry & $\rightarrow$ & $\begin{array}{l}\text { New types of polymers: “arborols," helical } \\
\text { polymetallocenes, etc. }\end{array}$ \\
\hline Physical chẹmistry & $\rightarrow$ & $\begin{array}{l}\text { More efficient photochemical systems; kinetics and } \\
\text { dynamics; chemistry under extreme conditions }\end{array}$ \\
\hline Organometallic chemistry & $\rightarrow$ & Catalytic polymers \\
\hline Electrochemistry & $\rightarrow$ & With monomers or polymers \\
\hline Solid-state chemistry & $\rightarrow$ & Polymeric ionic conductors \\
\hline Solid-state chemistry & $\leftarrow$ & Polymeric liquid crystals \\
\hline Atmospheric chemistry & $\rightarrow$ & Mechanisms of gas-phase polymerization \\
\hline
\end{tabular}


Future Directions of Research on Polymeric Materials

Table IV. Some ideas from (and to) the biological sciences

\begin{tabular}{|c|c|c|}
\hline Biological sciences & & Polymer science \\
\hline Molecular biology & $\rightarrow$ & Helicity, steric specificity \\
\hline Enzymes structure & $\rightarrow$ & Synthetic enzymes, catalysts, "biomimetic" polymers \\
\hline Base pairing & $\rightarrow$ & Specific functional sites in polymers \\
\hline Vesicles, liposomes & $\rightarrow$ & Polymeric analogs \\
\hline Aggregation phenomena & $\rightarrow$ & Gelation \\
\hline Primary structure & $\rightarrow$ & Periodic copolymers with many types of monomeric units \\
\hline Biotechnology & $\rightarrow$ & Monomers, polymers \\
\hline Merrified synthesis & $\leftarrow$ & Ion-exchange resins \\
\hline Biological materials & $\leftarrow$ & Materials science \\
\hline
\end{tabular}

Table V. Some ideas from (and to) metallurgy and ceramics science

\begin{tabular}{lll}
\hline \multicolumn{1}{c}{ Metallurgy and ceramics science } & & \\
\hline Phase transformations & $\rightarrow$ & Spinodal decomposition in polymers \\
Martensitic transformation & $\rightarrow$ & Epitaxial polymerization \\
Fracture, tribology & $\rightarrow$ & Mechanical behavior of polymers, incl. crazing \\
Defects: disclinations, grain boundaries & $\rightarrow$ & Interfaces in multicomponent polymers \\
Electron microscopy & $\rightarrow$ & Application to polymers \\
Surface analysis & $\rightarrow$ & Application to polymers \\
Epitaxy & $\rightarrow$ & Epitaxial growth in polymers \\
Positron annihilation & $\rightarrow$ & Voids and free volume \\
Glasses, amorphous metals & $\leftarrow$ Amorphous polymers \\
Free volume & $\leftarrow$ Physical aging \\
Ceramics synthesis & $\leftarrow$ Polymeric precursors \\
Sol-gel process & $\leftarrow$ Polycondensation \\
Modification of glass surfaces; & $\leftarrow$ Composites \\
$\quad$ inorganic-matrix composites & & \\
\hline
\end{tabular}

tenuous, but a few far-sighted scientists are beginning to venture into the interface between the two fields. As we strive to emulate biological systems by building higher specificity in function into synthetic polymers, by seeking yet more effective ways of controlling the steric structure of polymers, and by searching for new methods of controlling sequences of different monomeric units (beyond the three that seem to be the limit at this time), a new rapprochement of the biological sciences and polymer science seems inevitable. The emerging recognition that biological materials (e.g., muscle, bone, organs) are amenable to study by the methods of materials science will aid this process.

\section{Metallurgy and Ceramics Science}

The fields of metallurgy and ceramics science, both based on ancient industries, are in a state of vigorous rejuvenation. Polymer scientists are learning much from these fields, in such important areas as phase separations and transformations, fracture and fatigue behavior, tribology, the effect of grain boundaries and defects on properties, and the effective use of electron microscopy and surfaceanalytical techniques, which are far more advanced in these disciplines than in polymer science. ${ }^{8}$ Table $\mathrm{V}$ shows that the movement of ideas to and from polymer science is about equal. 
Table VI. Examples of modern instrumentation for polymer research (in approximately decreasing cost)

\begin{tabular}{ll}
\multicolumn{1}{c}{ Instrument } & \multicolumn{1}{c}{ Typical application } \\
\hline SANS & (Very expensive) \\
Synchrotron radiation & Molec. arrangement in crystals, networks \\
- Rutherford backscattering & Photolithography \\
- EXAFS & Crazing \\
Supercomputer & Ionomers \\
30T magnets & Previously unfeasible calculations \\
& NMR, liquid crystals \\
STEM, TEM, SEM & (Expensive) \\
Solid-state NMR & Studies at low dimensions \\
XPS, etc. & Macromolecular dynamics, networks \\
SAXS & Surface composition \\
Cloud chambers & Phase separations, crazing \\
Ultrafast spectroscopies & Kinetics of gas-phase polymerization \\
& Macromolecular dynamics \\
LAM-Raman spectroscopy & (Moderately expensive) \\
FTIR & Defects in crystals \\
Lasers & Surface analysis, hydrogen bonding, etc. \\
QELS & Photochemistry \\
Israelachvili apparatus & Diffusion in solutions, amorphous polymers \\
Microcomputer & Adsorption forces \\
Minicomputer & Faster calculations \\
& Operation of lab apparatus \\
\hline
\end{tabular}

\section{THE IMPACT OF NEW INSTRUMENTATION}

Much of modern science, including of course research on polymeric materials, is instrument-driven and perhaps instrumentlimited. ${ }^{8}$ The new instruments are both more powerful and allow measurements of a kind that was previously unfeasible. Access to these instruments is an absolutely essential requirement for research at the frontier of science. Because of their size, cost, and complexity, some instruments can be located only in a few, nationally maintained laboratories to which the scientists must travel. That is the case for the first of the three groups in Table VI, which divides instruments by approximate cost. All large instruments share the characteristic that they require a specialist for their optimal operation; thus, polymer researchers are becoming increasingly dependent on these specialists for their research.
Polymer science has been enormously aided by many new instruments. Let me cite a recent example. Using a Class VI computer, Suter was able to achieve the first calculation of bulk properties of a polymer (atactic polypropylene) from basic principles of atomistic behavior, rotational isomeric state theory, and long-range interactions. ${ }^{14}$ This extraordinary feat would not have been possible, even using a supercomputer, were it not for stagewise minimization to reduce the immense number of calculations. The results of these calculations agree remarkably with experimentally determined values of several properties of the bulk polymer.

\section{THE DEMANDS OF "HIGH- TECHNOLOGY” INDUSTRIES}

Polymers are increasingly being used in many of the most demanding industrial applications. ${ }^{15}$ They have several, well-recognized 
Table VII. Examples of polymeric materials used in "high-technology" applications

\begin{tabular}{ll}
\multicolumn{1}{c}{ Polymers } & \\
\hline Photodegradable polymers & Applications \\
Photopolymerizable systems & Photoresists \\
Conductive polymers, incl. ionic conductors & Photoresists \\
Magnetic polymers & Batteries \\
Ferroelectric polymers & Recording media \\
Structural composites & Transducers \\
Fibers & Space vehicles, aircraft, flywheels, automobiles \\
Matrix materials & \\
Liquid-crystalline polymers & \\
Thin films & Structural materials; nonlinear optics \\
Polyimides & Electronic devices; membranes \\
Adhesives & Electronic packaging \\
Biocompatible polymers & Rapid assembly \\
Oligomers & Prostheses, drug carriers, drugs \\
Polymeric coupling agents & Functional polymers \\
& Composites \\
\hline
\end{tabular}

advantages: a broad range of chemical structures coupled with increasingly precise control of the molecular architecture and morphology, ease of fabrication, and great versatility in the physical properties that are possible. A few examples of "high-technology" applications are given in Table VII; this important subject is dealt with in greater detail in the preceding paper, ${ }^{16}$ and in many others at this Conference.

The interest in polymers for "hightechnology" applications is widespread because it is tied directly to the future economic competitiveness of industries. In the United States, the NSF has provided several mechanisms for aiding collaborative research between universities and industrial organizations, including small businesses. Similar objectives are being pursued -although possibly by different mechanisms - in Japan (through the ERATO Fine Polymers Project directed by Professor Ogata), in France, Italy, and several other countries.

Research to meet the specialized needs of "high-technology industries". demands the utmost versatility in synthesis, as well as a profound understanding of such other sciences as physics, materials behavior, mechanics, biology, etc. Another important distinction is that polymers are simply one of many materials available to meet a given functional requirement. Thus, familiarity with a broad range of materials, whether they be polymeric or not, is essential.

\section{THE FUTURE OF POLYMER SCIENCE IN UNIVERSITIES}

It now remains for us to consider some of the anticipated consequences of these trends. In the author's view, they will be significant and will alter, in a profound way, what is now considered to be polymer science. The professional environment of the academic polymer scientist will also be affected.

The growing influence of the condensedmatter physicists is expected to drive polymer research increasingly closer to the field of physics. Similarly, the increasing involvement of talented chemists without prior history of contributing to the field of polymers is going to shift polymer science much closer to the mainstream of chemistry. A comparable statement can also be made for the growing commonality between polymer science and the other materials sciences, namely metallurgy and ceramics science. But the movement will not be one-way: many of the newcomers gravi- 


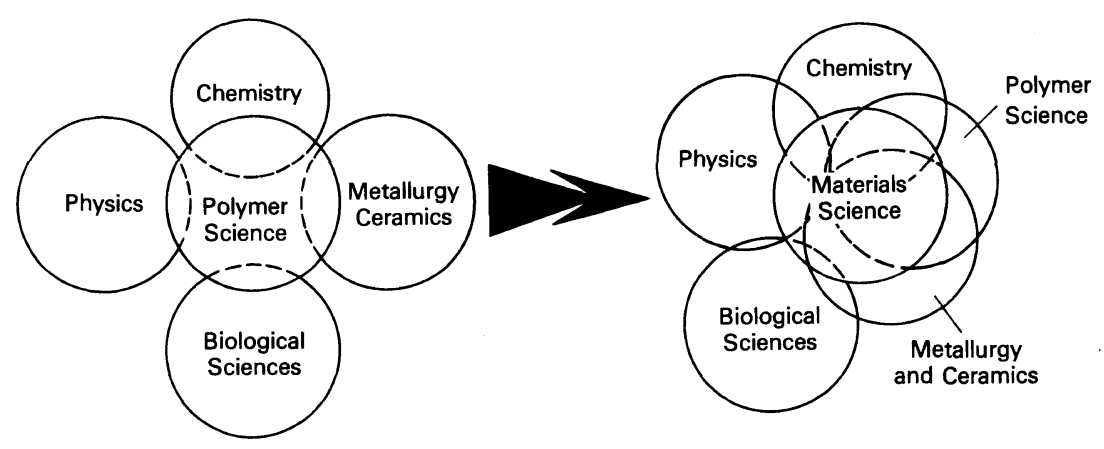

Figure 2. Convergence of several sciences to create the new discipline materials science.

tating toward polymer research are finding that success requires knowledge and insight of a kind that their prior background in physics, chemistry, or metallurgy does not provide. And, as has been shown above, polymer science has much to contribute to other sciences.

As the sciences dealing with materials get increasingly intermingled and mutually dependent, a new blend is emerging at their confluence: it is a fusion of polymer science and engineering, metallurgy and ceramics science, with components of chemistry and physics, mechanics, rheology, and, eventually, biology. That composite is the new discipline of materials science! This blending is illustrated in Figure 2. One consequence of this trend should be that few, if any, new departments of polymer science will be created in major American universities-but there should be many departments of materials science.

Because the science will be changing, we can expect that academic and professional organizations, and the way scientists carry out their research, will also be profoundly altered. Few individual scientists will be able to deal, alone, with the widening scope of materials research and the ever-increasing size, cost, and complexity of the powerful new instruments. Most will find that they lack the specialized knowledge to make a broad contribution to the diverse and difficult research on new high- performance materials. Many university scientists (but by no means all) will conclude that multidisciplinary groups are the best way to make progress in materials research - a path already encouraged by the government agencies funding academic research.

The polymer scientist will then become one specialist in a team comprised of several specialists working, in common, on broad problems. This cooperative mode of research, well known in industry, is already seen in American universities in the Materials Research Laboratories, Materials Research Groups, and Engineering Research Centers-all funded by the National Science Foundation. Table VIII shows a typical budget for a Materials Research Laboratory, compared with the budget for a NSF grant to a single senior investigator. Table IX illustrates the scope of a major research theme at such a laboratory; a variety of techniques is being applied to both scientific and technological issues.

In this multidisciplinary environment, the ties of the scientist to his original discipline will inevitably be weakened. This trend will force universities and scientific organizations to adapt or find themselves increasingly obsolete.

The Critical Role of Synthesis. Finally, the increasingly critical role of the synthesis specialist should be noted. In the new organization of research that has been described, he will be called upon not only to find still better 
Table VIII. Typical NSF budget for different kinds of research groups, in $\mathbf{k} \$$

\begin{tabular}{clc}
\hline Individual investigator & & Multidisciplinary group \\
\hline 40 & Salaries & 950 \\
1 professor, 2 students & & 11 professors, 43 students, 2 postdocs, \\
& & 16 technicians, 8 secretaries \\
5 & Materials & 320 \\
9 & Travel, misc. & 95 \\
23 & Equipment & 675 \\
\hline 80 & Overhead & 960 \\
\hline
\end{tabular}

Table IX. Scope of a typical major research theme at a NSF-funded Materials Research Laboratory

\begin{tabular}{c} 
Polymer interfaces \\
$\begin{array}{c}\text { Scientific issues } \\
\text { Preparation of novel interfaces } \\
\text { Conformations of constrained chains } \\
\text { Thermodynamics of interfaces } \\
\text { Interfacial electronic states } \\
\text { Dielectric behavior } \\
\text { Technological issues } \\
\text { Adhesion } \\
\text { Separations } \\
\text { Fabrication of electronic devices } \\
\text { Remote sensing } \\
\text { Colloid stability } \\
\text { Techniques } \\
\text { Theory } \\
\text { Synthesis } \\
\text { Spectroscopy } \\
\text { Microscopy } \\
\text { Electrophoresis } \\
\text { Calorimetry }\end{array}$ \\
\hline Measurements of electronic and dielectric properties \\
\hline
\end{tabular}

ways of controlling the molecular architecture, but also to use his utmost ingenuity to produce new functional materials - for a major theme of future research will be the discovery of new phenomena and new properties through the study of new polymeric materials. An additional, and increasingly crucial, role will be to prepare special materials needed by the physicist or the engineer in the team. This is illustrated in Figure 3. Both of these functions

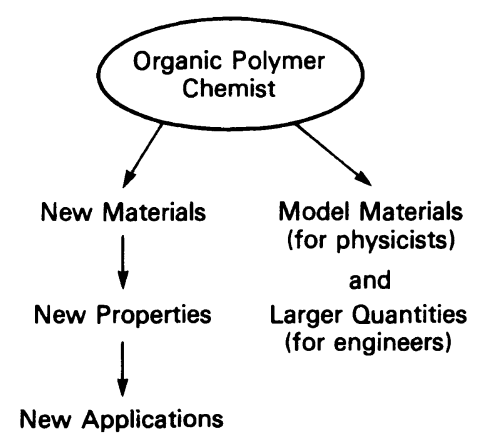

Figure 3. Expanded role for synthesis.

-one more creative, the other more supportive - should bring forth a renaissance of organic polymer chemistry.

Acknowledgments. The opinions presented are solely those of the author. The scientific advances described in this paper result from the efforts of many talented individuals; the specific citations should be taken to be merely illustrative.

\section{REFERENCES}

1. H. Morawetz, "Polymers: The Origins and Growth of a Science," John Wiley \& Sons, New York, N. Y., 1985.

2. "Polymer Science and Engineering: Challenges, Needs, and Opportunities," National Academy Press, Washington, 1981.

3. E. L. Thomas, D. B. Alward, D. J. Kinning, D. C. Martin, D. L. Handlin, Jr., and L. J. Fetters, Macromolecules, in press (1986).

4. T. Higashimura, M. Miyamoto, and M. Sawamoto, Macromolecules, 18, 611 (1985). 


\section{N. M. BIKAAES}

5. R. Faust and J. P. Kennedy, Polym. Bull., 15, 317 (1986).

6. A. J. Pennings, J. Smook, J. de Boer, S. Gogolewski, and P. F. van Hutten, Pure Appl. Chem., 55, 777 (1983).

7. S. Granick, S. Patel, and M. Tirrell, Nature, in press (1986).

8. "Trends and Opportunities in Materials Research," Publication SF-640, National Science Foundation, Washington, 1984.

9. P.-G. de Gennes, "Scaling Concepts in Polymer Physics," Cornell University Press, Ithaca, 1979.

10. P. J. Flory, "Principles of Polymer Chemistry," Cornell University Press, Ithaca, 1953.

11. "Opportunities in Chemistry," National Academy
Press, Washington, 1985.

12. D. A. Tomalia, H. Baker, J. Dewald, M. Hall, G. Kallos, S. Martin, J. Roeck, J. Ryder, and P. Smith, Polym. J., 17, 117 (1985).

13. G. R. Newkome, Z. Yao, G. R. Baker, V. K. Gupta, P. S. Russo, and M. J. Saunders, J. Am. Chem. Soc., 108, 849 (1986)

14. D. N. Theodorou and U. W. Suter, Macromolecules, 18, 1467 (1985); ibid., 19, 139 (1986); ibid., 19, 379 (1986).

15. N. M. Bikales, "Advanced Polymeric Materials for the Future," J. Polym. Sci., Symposium Issue Honoring H. Mark's 90th Birthday, in press.

16. G. Allen, Polym. J., 19, 1 (1987). 